\title{
Desarrollo calorimétrico del proceso de precipitación en aleaciones
}

\section{Cu-Co-Si}

\author{
E. Donoso*, A. Varschavsky*y G. Díaz* \\ Resumen Mediante calorimetría diferencial de barrido (DSC) se estudió el proceso de precipitación \\ de soluciones sólidas supersaturadas de tres aleaciones de $\mathrm{Cu}-\mathrm{Co}-\mathrm{Si}$, con composición de \\ cobalto constante. Evaluaciones entalpimétricas y estudios previos, revelaron que la \\ descomposición comienza con la precipitación de cobalto. El agrupamiento de átomos de \\ cobalto inicia la precipitación del silicio, formándose finalmente partículas de composición \\ estequiométricas de $\mathrm{Co}_{2} \mathrm{Si}$. Las fracciones volumétricas fueron determinadas en función de \\ la cantidad de cobalto presente en estas aleaciones. Se infiere que el superávit de átomos \\ de silicio retenidos en la solución aumenta la velocidad de reacción. Los parámetros \\ cinéticos fueron calculados a partir de un método basado en el formalismo de Mehl- \\ Johnson-Avrami (MJA). La energía de activación más baja obtenida, asociada con el \\ apilamiento de cobalto, se atribuyó a la contribución de las vacantes retenidas por temple.
}

Palabras clave Cobre. Cobalto. Silicio. Precipitación. Cinética. Difusión.

\section{Calorimetric development and study of precipitation processes in Cu-Co-Si alloys}

Abstract

Keywords

\begin{abstract}
Using differential scanning calorimetry (DSC) the precipitation processes of supersaturated solid solutions of three $\mathrm{Cu}-\mathrm{Co}-\mathrm{Si}$ alloys, with a constant cobalt composition, were studied. Thermograms, and previous studies, reveal that the decomposition begins with cobalt precipitation. Clustering of cobalt initiates the silicon precipitation, finally $\mathrm{Co}_{2} \mathrm{Si}$ stoichiometric particles are formed. Volume fractions are determined by the amount of cobalt present in these alloys. It is infered that surplus silicon atoms retained in the solution increase the reaction rate. Kinetic parameters were calculated by a method based in the Mehl-Johnson-Avrami (MJA) formalism. The lower activation energy obtained, associated with cobalt clustering is attributed to the contribution of quenched-in vacancies.
\end{abstract}

Copper. Cobalt. Silicon. Precipitation. Kinetics. Diffusion.

\section{INTRODUCCIÓN}

El desarrollo de aleaciones ternarias de alta resistencia está siendo motivo de variados estudios desde mediados de la década pasada. En la mayor parte de ellas, se fundamenta su resistencia mecánica en la formación de precipitados binarios y/o ternarios de extrema fineza, resistentes a ser cizallados por las dislocaciones, confiriéndole al material un elevado límite de fluencia. En el caso de las aleaciones de $\mathrm{Cu}-\mathrm{Co}-\mathrm{Si}$, el proceso de precipitación ha sido estudiado por resistividad eléctrica ${ }^{[1-3]}$, fuerzas termoeléctricas ${ }^{[2}$ y 3$]$ y calorimetría diferencial de ba- rrido $^{[3-6]}$. Estos últimos estudios han considerado el comportamiento mecánico de dichas aleaciones y, además, han dado información acerca de la nucleación y crecimiento de los precipitados estables $\mathrm{Co}_{2} \mathrm{Si}$. Trabajos anteriores de los autores de la presente investigación ${ }^{[7,8]}$ estudiaron el comportamiento a la fatiga de algunas aleaciones de $\mathrm{Cu}-\mathrm{Co}-\mathrm{Si}$ de composiciones cuasi binarias $\mathrm{Cu}-\mathrm{xCO}_{2} \mathrm{Si}$. Los principales objetivos del presente estudio son: caracterizar el proceso de precipitación del $\mathrm{CO}_{2} \mathrm{Si}$, a partir de soluciones supersaturadas de $\mathrm{Cu}-\mathrm{Co}-\mathrm{Si}$ con, aproximadamente, igual concentración de átomos de cobalto, pero con diferente superávit de

$\left({ }^{*}\right)$ Universidad de Chile, Facultad de Ciencias Físicas y Matemáticas, Dpto. de Ciencia de los Materiales, Casilla 1420, Santiago-Chile. e-mail: edonoso@cec.uchile.cl 
átomos de silicio con respecto a la composición estequiométrica de $\mathrm{Co}_{2} \mathrm{Si}$, y estimar la cinética de precipitación mediante el formalismo de MehlJohnson-Avrami en conjunto con un modelo de convolución.

\section{MATERIALES Y PROCEDIMIENTO EXPERIMEN- TAL}

Los detalles de la preparación de aleaciones similares pueden hallarse en trabajos anteriores de los autores $^{[4-6]}$. Después de un análisis químico, se encontró que las aleaciones contenían: a) aleación A: $\mathrm{Cu}-0,65$ at.\% Co-0,33 at.\% Si; b) aleación B: $\mathrm{Cu}-0,65$ at.\% Co-0,86 at.\% Si; c) aleación C: $\mathrm{Cu}-$ 0,66 at.\% Co-1,1 at.\% Si. Se puede notar que, la aleación A corresponde al cuasi-binario $\mathrm{Cu}-1$ at.\% $\mathrm{CO}_{2} \mathrm{Si}$.

\section{RESULTADOS Y DISCUSIÓN}

La figura 1 muestra los termogramas típicos para las tres aleaciones en estudio, en forma de capacidad calórica diferencial $\Delta \mathrm{C}_{\mathrm{p}}$ frente a temperatura, para diferentes velocidades de calentamiento $\mathrm{li}$ neal, $\phi$. Las curvas DSC muestran dos efectos exotérmicos sucesivos y traslapados, etapas 1 y 2 , en donde el traslape aumenta con el incremento de silicio. Estos, pueden ser asociados a la formación de precipitados de $\mathrm{Co}$ y $\mathrm{Co}_{2} \mathrm{Si}$, respectivamente ${ }^{[3-6]}$. A mayores temperaturas, ocurre una reacción endotérmica, etapa 3, que corresponde a la disolución de las partículas de $\mathrm{CO}_{2} \mathrm{Si}$, ya que el valor del calor de reacción, $\Delta \mathrm{H}$, es aproximadamente igual al de la etapa 2 para una misma velocidad de calentamiento, $\phi$, e igual aleación. Los calores de reacción, áreas bajo las curvas de $\mathrm{DC}_{\mathrm{p}}$ vs. $\mathrm{T}$, se muestran en la Tabla I. Se puede observar una pequeña dismi-

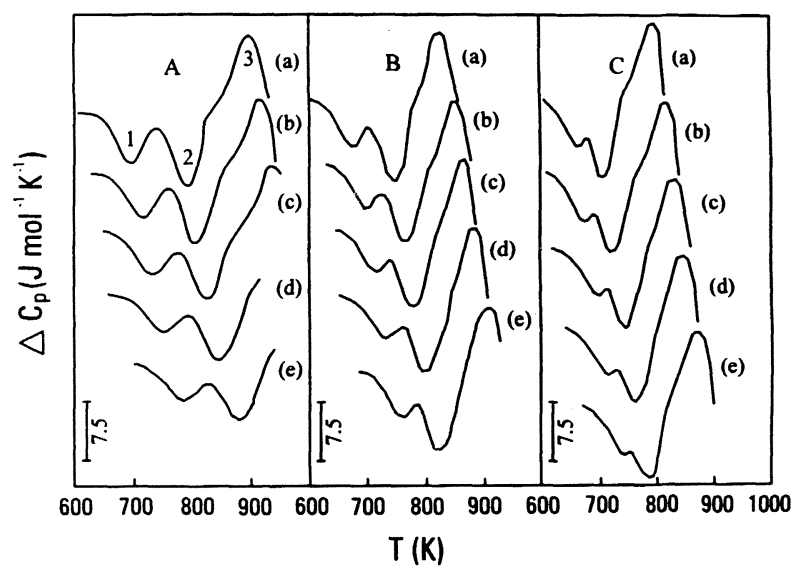

Figura 1. Trazas DSC para las aleaciones $A, B$ y $C$ donde se muestran los tres efectos calóricos principales. (a) 0,03 $\mathrm{ks}^{-1}$, (b) $0,08 \mathrm{ks}^{-1}$, (c) $0,16 \mathrm{ks}^{-1}$, (d) $0,33 \mathrm{ks}^{-1}$, (e) $0,83 \mathrm{ks}^{-1}$.

Figure 1. DSC traces for alloys $A, B$ and $C$ where the three main heat effects are shown. (a) $0.03 \mathrm{ks}^{-1}$, (b) $0.08 \mathrm{ks}^{-1}$, (c) $0.16 \mathrm{ks}^{-1}$, (d) $0.33 \mathrm{ks}^{-1}$, (e) $0.83 \mathrm{ks}^{-1}$.

nución en el valor de $\Delta \mathrm{H}$ asociado a todas las etapas, a medida que $\phi$ aumenta. Este efecto no es considerable, pero puede ser atribuido en todos los casos al hecho de que el calentamiento ocurre en un rango de temperatura mayor si se relaciona con el solvus de $\mathrm{Co}$ y $\mathrm{CO}_{2} \mathrm{Si}$ para valores de $\phi$ crecientes.

Si se evalúa la entropía molar de disolución a partir de $\Delta \mathrm{S}_{\mathrm{p}}=\Delta \mathrm{H}_{\mathrm{p}} / \mathrm{T}_{03}$, donde $\Delta \mathrm{H}_{\mathrm{p}}\left(221,6 \mathrm{~kJ} \mathrm{~mol}^{-1}\right)$ es la entalpía de disolución de los precipitados ${ }^{[5 \text { y } 6]}$, y $\mathrm{T}_{03}(826 / 765 / 716 \mathrm{~K}$, para a $\phi \rightarrow 0$, en las aleaciones $A / B / C$, respectivamente), es la temperatura de inicio del pico de disolución, se obtienen valores de $\Delta \mathrm{S}_{\mathrm{p}}=0,27 / 0,28 / 0,32 \mathrm{Jmol}^{-1} \mathrm{~K}^{-1}$ para las aleaciones $\mathrm{A} / \mathrm{B} / \mathrm{C}$. El pequeño aumento en $\Delta \mathrm{S}_{\mathrm{p}}$ es el reflejo de una mayor cantidad de orden interno que exhiben aquellos precipitados correspondientes a mayores concentraciones de silicio, en acuerdo con su menor tamaño. Este comportamiento se encuentra en

Tabla I. Calores de reacción DH involucrados a las diferentes etapas (J mol $\left.{ }^{-1}\right)$

Table I. Reaction heats involved in the different stages $\left(J \mathrm{~mol}^{-1}\right)$

\begin{tabular}{cccccccccc}
\hline$\phi$ & \multicolumn{3}{c}{ Aleación A } & \multicolumn{3}{c}{ Aleación B } & \multicolumn{3}{c}{ Aleación C } \\
\hline (Ks-1) & etapa 1 & etapa 2 & etapa 3 & etapa 1 & etapa 2 & etapa 3 & etapa 1 & etapa 2 & etapa 3 \\
0,033 & 339 & 585 & 580 & 342 & 583 & 577 & 335 & 585 & 579 \\
0,083 & 333 & 577 & 570 & 335 & 575 & 568 & 328 & 581 & 575 \\
0,167 & 325 & 563 & 558 & 330 & 567 & 563 & 318 & 568 & 561 \\
0,333 & 320 & 554 & 547 & 323 & 556 & 555 & 315 & 558 & 553 \\
0,833 & 315 & 545 & 538 & 318 & 548 & 544 & 308 & 546 & 544 \\
\hline
\end{tabular}

* Los datos representan el promedio de 5 ensayos calorimétricos 
concordancia con una menor probabilidad de falla de apilamiento en partículas de menor tamaño.

El hecho que el calor de reacción, para un mismo valor de $\phi$, en las etapas 1,2 y 3 sea, prácticamente, el mismo en las tres aleaciones, implica que la concentración de cobalto controla la cantidad de $\mathrm{Co}_{2} \mathrm{Si}$ precipitada ya que ésta es igual para todas las aleaciones. Puesto que la aleación $\mathrm{A}$ es el cuasi-binario $\mathrm{Cu}-1$ at. $\% \mathrm{CO}_{2} \mathrm{Si}^{[1-3]}$, la cantidad de silicio remanente a esta concentración permanece en solución. Se puede señalar que este efecto ocurre siempre que haya suficiente silicio para formar el compuesto $\mathrm{CO}_{2} \mathrm{Si}$.

Por otra parte, a partir de las trazas calorimétricas se puede evaluar la fracción volumétrica de las partículas a partir de $\mathrm{V}_{\mathrm{f}}=\left(\Delta \mathrm{H}_{3} / \Delta \mathrm{H}_{\mathrm{p}}\right) \times\left(\rho_{\mathrm{a}} / \rho_{\mathrm{p}}\right)$, donde, $\Delta \mathrm{H}_{3}$ es el calor de reacción de la etapa 3 , evaluada por extrapolación cuando $\phi \rightarrow 0$ y $\rho_{\mathrm{a}}$ y $\rho_{\mathrm{p}}$ son las densidades de las aleaciones y precipitados, respectivamente. Con los valores de $\Delta \mathrm{H}_{3}=$ $584 / 579 / 582 \mathrm{~J} \mathrm{~mol}^{-1}, \rho_{\mathrm{a}}=8,94 / 8,92 / 8,89 \mathrm{~kg} \mathrm{~m}^{-3}$ para las aleaciones $\mathrm{A} / \mathrm{B} / \mathrm{C}$ respectivamente, se obtiene aproximadamente $V_{f}=3,5 \times 10^{-3}$ para las tres aleaciones, valor que concuerda con los resultados previos dados con respecto al control que ejerce la concentración de cobalto en el proceso de precipitación.

Las energías de activación, E, de las diferentes reacciones fueron calculadas a partir de un método de Kissinger modificado ${ }^{[9]}$ :

$$
\ln \left(\frac{T_{p}^{2}}{\phi}\right)=\frac{E}{R T_{p}}+\ln \left(\frac{E}{R k_{o}}\right)
$$

donde, $T_{p}$ es la temperatura del pico, $k_{o}$ es el factor pre-exponencial y $\mathrm{R}$ es la constante de los gases. Entonces, $\mathrm{E}_{\mathrm{y}} \mathrm{k}_{\mathrm{o}}$ pueden ser evaluados a partir de gráficos $\ln \left(\mathrm{T}_{\mathrm{p}}^{2} / \phi\right)$ vs. $1 / \mathrm{T}_{\mathrm{p}}$, los cuales se muestran en la figura 2. Los valores de $\mathrm{E} \mathrm{y} \mathrm{k}_{\mathrm{o}}$, indicados en la Tabla II, son independientes del modelo cinético escogido siempre que $T_{p}$ sea la temperatura del pico $^{[9]}$. Los valores de las energías obtenidas resul-

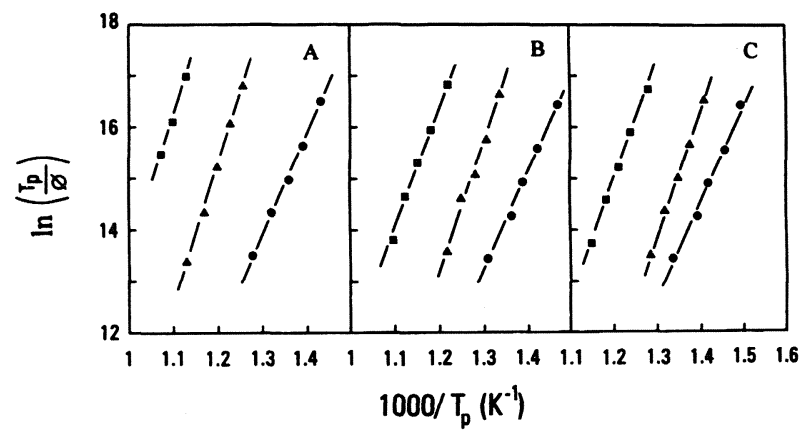

Figura 2. Gráfico de Kissinger modificado para las diferentes etapas en las aleaciones $A, B$ y $C$. ( $(0)$ etapa $1,(\mathbf{A})$ etapa 2 , ( $)$ etapa 3 .

Figure 2. Modified Kissinger plots for the different DSC stages in alloys A, B and C. (O) stage 1, (A) stage 2, ( stage 3 .

taron levemente mayores que los calculados por Lendvai et al. ${ }^{[3]}$.

Usando las correlaciones de Brown y Ashby ${ }^{[10]}$ y de Shi et al..$^{[1]}$, se estimó la energía de activación para la difusión del cobalto en cobre, $\mathrm{E}_{\mathrm{Cu}}{ }^{\mathrm{Co}}$, en $203,5 \mathrm{~kJ} \mathrm{~mol}^{-1}$, la cual resultó ser mucho más alta que el valor calculado para la etapa 1 . Este hecho puede ser atribuido a la fuerte contribución de las vacantes introducidas por temple, con una energía de activación para la migración de aproximadamente la mitad del valor de la energía $\mathrm{E}_{\mathrm{Cu}}{ }^{\mathrm{Co}}$. Como es de esperar, las energías de activación obtenidas para la etapa 1 no varían entre una aleación y otra, ya que la composición de cobalto es la misma y la difusión de silicio no interviene en esta etapa. A partir de los datos de la bibliografía ${ }^{[10}$ y 11], se estimó para la difusión de silicio en cobre un valor de $\mathrm{E}_{\mathrm{Cu}}^{\mathrm{Si}}=202 \mathrm{~kJ} \mathrm{~mol}^{-1}$. Se puede demostrar que las vacantes introducidas por el temple juegan un importante papel en la precipitación de cobalto, su efecto de aniquilación proporciona una contribución insignificante en el calor de reacción $\mathrm{DH}_{1}$. La fracción de $\mathrm{DH}_{1}$ asociado con la aniquilación de vacancias, $f_{v}$, puede ser evaluada a partir de:

Tabla II. Energías de activación y factores pre-exponenciales para las tres aleaciones

Table II. Activation energies and pre-exponential factors for the three alloys

\begin{tabular}{lccccccccc}
\hline & \multicolumn{3}{c}{ Aleación A } & \multicolumn{3}{c}{ Aleación B } & & \multicolumn{2}{c}{ Aleación C } \\
\cline { 2 - 10 } & etapa 1 & etapa 2 & etapa 3 & etapa 1 & etapa 2 & etapa 3 & etapa 1 & etapa 2 & etapa 3 \\
\hline $\mathrm{E}$ & & & & & & & & & \\
$\left(\mathrm{kJ} \mathrm{mol}{ }^{-1}\right)$ & 155,6 & 208,2 & 198,2 & 153,8 & 203,7 & 194,4 & 152,1 & 197,2 & 189,8 \\
$\operatorname{kox} 109(\mathrm{~s}-1)$ & 0,66 & 430 & 6,5 & 0,31 & 50 & 3,1 & 0,12 & 15 & 6,0 \\
\hline
\end{tabular}




$$
f_{v}=\exp \left(\frac{\Delta S_{k}}{R}\right) \exp \left(-\frac{\Delta H_{f}}{R T_{q}}\right) \frac{\Delta H_{f}}{\Delta H_{1}}
$$

donde, $\Delta \mathrm{H}_{\mathrm{f}}\left(106,4 \mathrm{~kJ} \mathrm{~mol}^{-1}\right)^{[12]}$ es la energía de formación de la vacancia, $\Delta \mathrm{S}_{\mathrm{k}}=5,76 \mathrm{~J} \mathrm{~mol}^{-1} \mathrm{~K}^{-1}[13]$, $\Delta \mathrm{H}_{1}=340 \mathrm{~J} \mathrm{~mol}^{-1}$ y $\mathrm{T}_{\mathrm{q}}(1.073 \mathrm{~K})$ es la temperatura de temple. El valor promedio obtenido para $f_{v}$ es de $4,1 \times 10^{-3}$, el cual resultó insignificante. Para la etapa 2 , puesto que la energía de activación aparente es un valor ponderado entre aquella del $\mathrm{Si}$ en $\mathrm{Co}$ y $\mathrm{Si}$ en $\mathrm{Cu}$, y puesto que el volumen de la partícula de $\mathrm{Co}_{2} \mathrm{Si}$ es pequeño si se compara con la correspondiente al seno del material, las energías de activación medidas deben ser similares a aquellas de Si en Cu.

El análisis cinético para las etapas 1 y 2 en las tres aleaciones, se realizó empleando la ecuación usual de Mehl-Johnson-Avrami (MJA), utilizada para reacciones heterogéneas, bajo condiciones no isotermales: $\boldsymbol{y}=1-\exp \left[-\left(\mathrm{k}_{\mathrm{o}} \theta\right)^{\mathrm{n}}\right]$; donde, $\boldsymbol{y}$ es la fracción reaccionada, $\mathrm{k}_{\mathrm{o}}$ es un factor pre-exponencial verdadero ${ }^{[14]}$ y $\theta\left(T^{2} R / \phi E\right.$ exp[-E/RT]) es el tiempo reducido ${ }^{[15]}$. Para el análisis de los picos traslapados se usó un modelo de convolución basado en el formalismo de MJA propuesto por Borrego y González-Doncel ${ }^{[16,17]}$ :

$$
\dot{y}=n k_{o}^{n}\left(\frac{T^{2} R}{\phi E}\right)_{-}^{n-1} \exp \left(-\frac{n E}{R T}\right) \exp \left[-k_{o}^{n}\left(\frac{T^{2} R}{\phi E}\right)^{n} \exp \left(-\frac{n E}{R T}\right)\right]
$$

y

$$
\Delta \dot{\mathrm{H}}_{\mathrm{T}}=\Delta \dot{\mathrm{H}}_{1}+\Delta \dot{\mathrm{H}}_{2}=\mathrm{A}_{1} \dot{\mathrm{y}}_{1}+\mathrm{A}_{2} \dot{\mathrm{y}}_{2}
$$

donde, el área total bajo las curvas traslapadas correspondientes a las etapas 1 y 2 , es $A=A_{1}+A_{2}$. Mediante el ajuste de las ecuaciones (3) y (4) con los valores de los calores experimentales obtenidos de las trazas calorimétricas, y usando las energías de activación aparente para las etapas 1 y 2 y sus respectivos factores pre-exponenciales (Tabla II), se obtienen los valores de los exponentes de la ecuación de MJA y los valores de $A_{1}$ y $A_{2}$. Puesto que el flujo calórico por unidad de masa de la muestra dado por, $\Delta \dot{H}=\Delta C_{p} \phi / M_{p}$, en donde $\mathrm{MW}_{\mathrm{p}}$ es el peso molecular del precipitado $\left(58,93 \times 10^{-3}\right.$ y $48,65 \times 10^{-3} \mathrm{~kg} \mathrm{~mol}^{-1}$, para el Co y el $\mathrm{CO}_{2} \mathrm{Si}$, respectivamente), se pueden obtener los calores de reacción asociados a las etapas 1 y 2 . Los ajustes a las curvas experimentales para la aleación $\mathrm{A}$, usando $\phi=0,17 \mathrm{ks}^{-1}$, donde, $\mathrm{A}_{1}=320 \mathrm{~J} \mathrm{~mol}^{-1} \mathrm{y}$
$\mathrm{A}_{2}=569 \mathrm{~J} \mathrm{~mol}^{-1}$, se muestran en la figura 3 . En esta figura se dibujan la curva experimental, la curva ajustada y las curvas individuales correspondientes a cada etapa. Se obtuvieron curvas similares para las otras dos aleaciones, las cuales por razones de espacio no se muestran aquí. El mejor ajuste se obtuvo con $\mathrm{n}_{1}=1,13 / 1,16 / 1,14$ y $\mathrm{n}_{2}=1,48 / 1,58 / 1,51$, para las aleaciones $A, B$ y C. El valor de $n_{1}$ es indicativo de un proceso de nucleación y crecimiento a partir de una solución sólida $(n=1)^{[15]}$, aunque cierta precipitación puede ocurrir durante el temple en tanto que el valor de $\mathrm{n}_{2}$ es indicativo de un proceso de crecimiento a partir de núcleos preexistentes de tamaño no despreciable $(n=1,5)^{[18]}$.

En un trabajo previo de los autores ${ }^{[5]}$, se ha demostrado que, para una aleación cuasi-binaria $\mathrm{Cu}-1$ at.\% $\mathrm{Co}_{2} \mathrm{Si}$ (aleación $\mathrm{A}$ ), la cinética de disolución (etapa 3 ) se puede describir por $F(y)=1-(1-y)^{2 / 3}=$ $\mathrm{k}_{\mathrm{o}}{ }^{*} \theta$, donde $\mathrm{k}_{\mathrm{o}}{ }^{*}$ es una constante y $\theta$ es el tiempo reducido, definido anteriormente. De los gráficos $\mathrm{F}(\mathrm{y})$ vs. $\theta$, mostrados en la figura 4 para $\phi=0,033$ $\mathrm{ks}^{-1}$, se obtienen los valores de $\mathrm{k}_{\mathrm{o}}{ }^{*}$ iguales a $1,4 \times 10^{9} / 2 \times 10^{9} / 3 \times 10^{9} \mathrm{~s}^{-1}$, para las aleaciones $\mathrm{A} / \mathrm{B} / \mathrm{C}$. De la figura 4 , se observa claramente que el comportamiento de la disolución del $\mathrm{Co}_{2} \mathrm{Si}$ es ajustable al modelo cinético utilizado para las tres aleaciones en estudio, ya que resultaron ser rectas de pendientes $\mathrm{k}_{\mathrm{o}}{ }^{*}$. Además, se puede constatar que estos valores de $\mathrm{k}_{\mathrm{o}}{ }^{*}$ son del mismo orden de magnitud que aquellos obtenidos a partir del modelo

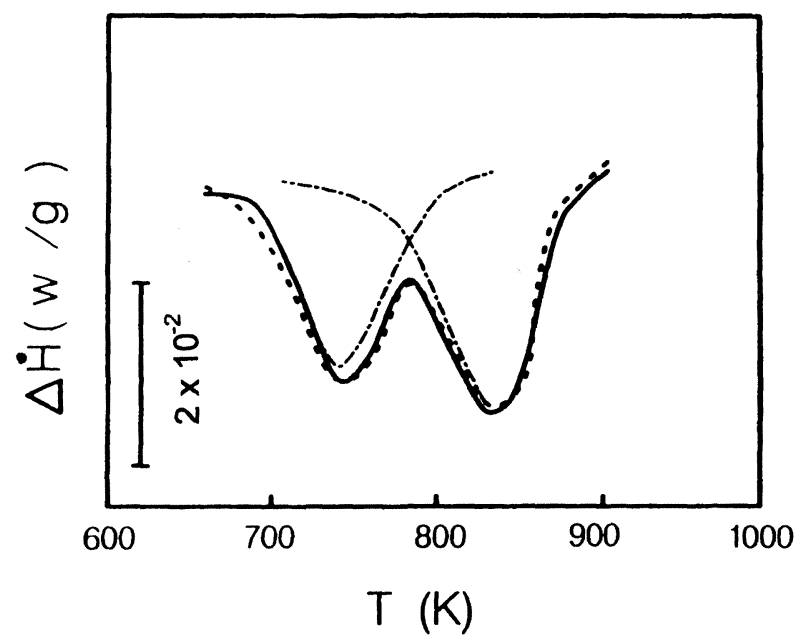

Figura 3. Flujo calórico frente a la temperatura de experiencias DSC af $=0,167 \mathrm{ks}^{-1}$, para la aleación A. ( ) curva experimental, (- ) curva ajustada, (- - - curvas individuales de las etapas 1 y 2.

Figure 3. Heat flow vs. temperature from DSC experiments at $f=0.167 \mathrm{ks}^{-1}$, for Alloy A. I ) experimental curve, (l adjusted curve, (- - individual curves of the stage 1 and 2. 


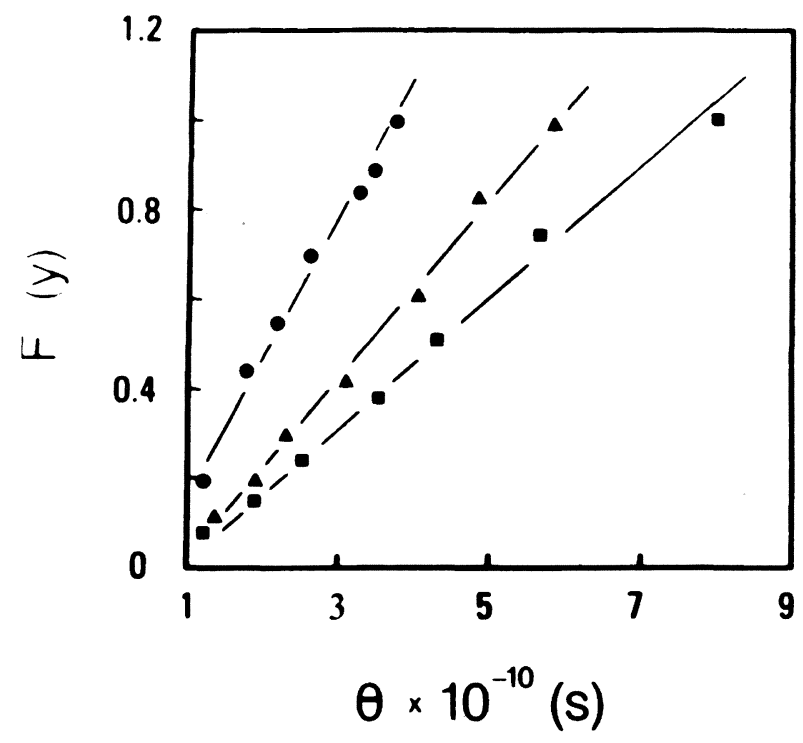

Figura 4. Gráficos de la función cinética integrada $F(y)=1$ $(1-y)^{2 / 3}$ frente a q correspondiente a la disolución de las partículas en las tres aleaciones. $(\square)$ aleación $A,(\boldsymbol{A})$ aleación $\mathrm{B}$ y $(0)$ aleación $\mathrm{C}$.

Figure 4. Plots of the integrated kinetic function $F(y)=1-(1-$ $y)^{2 / 3}$ vs. $q$ corresponding to particle dissolution in the three alloys. (ロ) alloy $A,(\Delta)$ alloy $B$ and (O) alloy C.

de Kissinger. Tales resultados permiten sugerir que los valores obtenidos a través de la ecuación (1) son independientes del modelo cinético escogido, como puede esperarse cuando se usa la temperatura del pico, $T_{p}$, en esta ecuación.

\section{CONCLUSIONES}

Los resultados anteriores permiten concluir lo siguiente:

- de acuerdo con los termogramas se puede inferir que, los precipitados de $\mathrm{Co}_{2} \mathrm{Si}$ se forman (etapa 2) por difusión de silicio en los apilamientos precursores de cobalto (etapa 1). Estas partículas de cobalto nuclean y crecen a partir de la solución sólida, después de templar y realizar un calentamiento lineal de las muestras.

- El silicio que excede a la composición estequiométrica del $\mathrm{Co}_{2} \mathrm{Si}$ permanece en solución, pero una concentración inicial mayor incrementa, notablemente, la nucleación de las partículas, obteniéndose una estructura final más fina.

- La reacción endotérmica (etapa 3) corresponde, inequívocamente, a la disolución de las partículas de $\mathrm{Co}_{2} \mathrm{Si}$. La fracción volumétrica está determinada por la cantidad de cobalto presente.

- Los parámetros cinéticos fueron determinados usando el formalismo de Mehl-Johnson-Avrami bajo condiciones no isotermales, en conjunto con un modelo de convolución basado en el mismo formalismo. Por último, la cinética de disolución de los precipitados se ajusta perfectamente bien a una ley cinética de difusión tridimensional.

\section{Agradecimientos}

Los autores desean agradecer al Fondo Nacional de Desarrollo Científico y Tecnológico (FONDECYT), Proyecto $N^{\circ} 1040795$ por el apoyo financiero, y a la Facultad de Ciencias Físicas y Matemáticas de la Universidad de Chile, por las facilidades otorgadas para desarrollar esta investigación.

\section{REFERENCIAS}

[1] B. Albert, Z. Metallk. 75 (1985) 475-478.

[2] B. Albert, Z. Metallk. 75 (1985) 528-531.

[3] J. Lendvai, T. Ungár, I. Kovács y B. Albert, J. Mater. Sci. 23 (1988) 4.059-4.066.

[4] E. Donoso, Rev. Metal. Madrid 37 (2001) 492-498.

[5] A. Varschavsky y E. Donoso, J. Thermal Anal. Cal. 68 (2002) 231-241.

[6] A. Varschavsky y E. Donoso, Mater. Lett. 57 (2003) 1.266-1.271.

[7] A. Varschavsky y E. Donoso, Mater. Lett. 15 (1992) 207-211.

[8] A. Varschavsky y E. Donoso, Anales del III Congreso Iberoamericano de Ingeniería Mecánica, Septiembre 1997, La Habana-Cuba, v. I, pp. 119-124.

[9] E.J. Mittemeijer, Lui Cheng, J. Van der ShaAf, C.M. Brakman y B.M. KorevaAR, Metall. Trans. 19A (1988) 925-932.

[10] A.M. Brown y M.F. Ashby, Acta Metall. 28 (1980) 1.0851.101.

[11] FJ. ShI, T.G. NieH y Y.T. Chou, Scripta Mater. 43 (2000) 265-267.

[12] E. Donoso y A. Varschavsky, J. Thermal Anal. 45 (1995) 1.419-1.435.

[13] A. Varschavsky y M. PilleuX, Mater. Letts. 17 (1993) 364-368.

[14] A. Varschavsky, Thermochim. Acta 203 (1992) 391-417.

[15] M.E. Brown y A.K. GalWey, Thermochim. Acta 387 (2002) 173-183.

[16] A. Borrego y G. GonzÁlez-Doncel, Mater. Sci. Eng. A245 (1998) 10-18.

[17] A. Borrego y G. González-Doncel, Mater. Sci. Eng. A276 (2000) 292-295.

[18] J.W. Christian, The theory of transformation of metals and alloys, $2^{\circ}$ Ed., Pergamon Press, England, 1971, p. 534. 